\title{
Sexual Harassment in the Field of Sexuality Research
}

\author{
Debby Herbenick ${ }^{1} \cdot$ Sari M. van Anders ${ }^{2} \cdot$ Lori A. Brotto $^{3} \cdot$ Meredith L. Chivers $^{4} \cdot$ Sofia Jawed-Wessel ${ }^{5}$. \\ Jayleen Galarza ${ }^{6}$
}

Received: 18 July 2018 / Accepted: 17 January 2019 / Published online: 1 February 2019

(c) Springer Science+Business Media, LLC, part of Springer Nature 2019

\section{Introduction}

Our field has a problem with sexual harassment, and we need to talk about it. Though sexual harassment is currently at the forefront of discussions taking place within major social movements, professional societies, and disciplines (see, for example, Clancy, Nelson, Rutherford, \& Hinde, 2014; Dzau \& Johnson, 2018; National Academies of Sciences, Engineering, and Medicine, 2018), the discipline of sexuality research has-to this point — been largely absent from these discussions. There are some exceptions, however, with a few sexuality researchers in sociology, psychology, and gender studies among those who have faced or made public accusations or formal reports alleging sexual misconduct or harassment (e.g., Flaherty, 2018; Grollman, 2018; Mondon, 2018). With this Guest Editorial, we aim to begin that discussion, articulating that \#TimesUp too in sexuality research, and present a collective united front against sexual harassment in our field and workplaces.

Our goal in this Guest Editorial is to articulate: (1) the scope of the problem of sexual harassment within our fields, especially sexuality research, including its consequences; (2) the

Drs. Debby Herbenick and Sari M. van Anders are co-first authors.

Debby Herbenick

debby@indiana.edu

1 Department of Applied Health Science, PH 116, Indiana University School of Public Health, Bloomington, IN 47405, USA

2 Departments of Psychology and Gender Studies; Program in Neuroscience, Queens University, Kingston, ON, Canada

3 Department of Obstetrics and Gynecology, University of British Columbia, Vancouver, BC, Canada

4 Department of Psychology, Queens University, Kingston, ON, Canada

5 Department of Health and Kinesiology, University of Nebraska, Lincoln, NE, USA

6 Department of Social Work, Shippensburg University, Shippensburg, PA, USA gendered basis of sexual harassment; (3) the exacerbation of these experiences for people of color and those in lower positions of power, including students and/or other minoritized social locations; and (4) suggestions toward stopping sexual harassment within sexuality professions, including sexuality research. While sexual harassment can occur between professionals and their clients, patients, and research participants, we will focus here on sexual harassment within research, academic, and professional spaces. In doing so, we draw on our own experiences and those of colleagues who have shared their experiences with us as well (either anonymized/grouped or with their permission). As we all live and (mostly) work in North America, we note the cultural limitations of our perspectives.

\section{Sexual Harassment in Context}

\section{What Sexual Harassment Is and Is Not}

Historically, sexual harassment was defined by men, with those targeted largely absent from the power structures that allow phenomena to be defined and acted upon. This power imbalance works in concert with majority group members who perpetrate other forms of group-based injustice, like White people deciding what is or isn't anti-Black racism or settler colonialists deciding what is or isn't anti-indigeneity. Those who experience injustice have always pushed back, and societal discussions about sexual harassment are a visible example of this right now. Thus, while some may argue that claims of sexual harassment are largely overblown, hysterical, censorious, or simple "misunderstandings," those of us who have experienced sexual harassment are inserting ourselves into a conversation that should have centered the voices of those affected from the start.

What is sexual harassment? Although its name might suggest otherwise, sexual harassment is a form of gender-based maltreatment or violence that may or may not be sexual and may or may not manifest as frank sexual overtures or acts. Sexual harassment includes, and is, gender-based harassment (see National Academies of Sciences, Engineering, and Medicine, 
2018; Schulz, 2018). To be more specific, sexual harassment may be "sexual," as with unwanted sexual attention or requests to engage in sexual acts. However, sexual harassment is more often gender-based, or "nonsexual." This includes derogating someone on the basis of their gender/sex or violations of their gender/sex norms, as with sexual minorities, nonsexual heterosexual men, or sexually agentic women, etc., and gender-based insults, jokes, and discrimination. Sexual harassment may be physical or verbal, in-person or electronic, isolated or repeated, or occur in groups or one-on-one. In our experience, sexual harassment can also be reflected in colleagues seeing/treating women or feminine people (especially young ones) at conferences as only one of two things: potential sexual partners or irrelevant.

Though sexual harassment perpetrated by heterosexual men against heterosexual women typically receives the most attention, sexual harassment is also perpetrated by and against people in a variety of minoritized social locations. As just a few examples (after all, anyone can harass or be harassed), some white women sexualize or exoticize women of color, some gay men sexually harass bisexual or other gay men, some cisgender lesbians undermine trans women, and so on. Indeed, because sexual harassment involves attempts to gain power through policing others' gender, many individuals who themselves are suspected of violating gender norms access power by policing others, as when some gay men sexually harass heterosexual women.

Sexual harassment is different from consensual sexual interactions and flirtations that occur between adults. We acknowledge that consensual sexual interactions can include power differentials and enjoyable ways that people play with power. Indeed, sexuality researchers are adults who are able to choose, consent to, and engage in sexual interactions with others, including colleagues. Yet we cannot deny how power imbalances and precarious social positions make choice and consent murky at times. In the context of power imbalance, such as senior/junior colleagues and professor/student relationships, consent is not always as simple as "doing what one wants" but may include concerns about consequences if one rejects advances from someone with more real or perceived power. Some of us (and you!) are or have been editors, society officers, program committee chairs, members of hiring committees, student advisors, clinicians, and/or respected or admired authorities. Our actions carry all the weight of our positions of power, such that some people may reasonably fear negative repercussions should they wish to reject unwanted attention or address someone's problematic behavior.

\section{Recent Publicized Examples of Sexual Harassment in the Media}

From Bill Cosby to Harvey Weinstein, Bill O'Reilly to Roger Ailes, Jian Ghomeshi to Kevin Spacey, recent examples of celebrities' alleged sexual harassment or violence show that it is largely described as being committed by men and most often against women. It occurs in variable ways, including coercion, persistence in the face of repeated rejection, abuses of power, threats, and even physical incapacitation, physical violence, and rape.

What has been astonishing to some, while long-known to others, is that (1) the sexual harassment is an open secret, with many harassers known to numerous people, (2) many people and levels of administration supported the harassers while silencing or undermining the victims, (3) the women and other people who have gone public have been widely vilified and harassed, and (4) the harassers have by and large experienced no consequences, and certainly almost no legal ones. Indeed, as victims know that they are unlikely to be believed, going public is one of the least likely options. Going public, including to judicial or official sources when deemed appropriate, often only feels available to those who occupy extreme positions of privilege or when sufficient numbers of victims come together to speak out against an alleged perpetrator, as with the cases involving Dr. Larry Nassar or Harvey Weinstein, or with social movements such as \#YesAllWomen and \#MeToo.

Each of us who has co-authored the present Guest Editorial has experienced sexual harassment within our field; none of us have named the harassers publicly, though we have made other efforts. Some of us have named our harassers in other ways (e.g., by reporting to our advisors, mentors, or programs) or spoken to the harassers directly (by explaining the situation and asking for changes in behavior). In the majority of our experiences, however, people have relied on sharing information through informal "whisper networks" as a way to support or protect others.

Choosing to come forward means risking reprisal, disbelief, and further trauma. After already enduring the humiliation of sexual harassment, many are not in a position to make themselves more psychologically vulnerable or further compromise their physical safety. In an academic context, we face the real fear that coming forward will compromise our careers. Some of us, even as established academics, have feared personal persecution and negative impact on our careers. This often kept us silent, in some cases for many years. For some, we did not initially recognize certain behaviors as sexual harassment, despite ourselves being embedded in the research, education, and/or clinical practice related to sexual harassment and abuse. One of us was told directly by their harasser, a person with significant academic power over them, that they should never disclose the incidents in question to others in our field, with the understood unspoken message being that it would tarnish her reputation (not his). As is typical of rape culture, instead of the perpetrator being subject to just consequences for their actions, the victim is blamed and further persecuted. When we do choose to come forward, the reception is rarely supportive, and often includes questioning that is either irrelevant or suggests culpability. Despite these challenges, some of us do come forward, 
working for change to help and protect others, hoping that, by reporting, others might not have to suffer through what we did. By writing this Guest Editorial, and starting this conversation within our field, this is what we hope to do.

\section{Sexual Harassment in Academic and Research Environments}

Sexual harassment is a ubiquitous issue permeating our society, including academic cultures, as our experiences clearly indicate. Yet, there are academic contexts within which sexual harassment is more or less likely to occur. Some contexts are more public or regulated than others (e.g., a classroom vs. a professor's office), although some of us have experienced frank public sexual harassment even in classroom settings. Many researchers and professors see themselves as experts in topics-including sexual harassment- that go far beyond their actual area of expertise, knowledge, or lived experience. In our experiences, some sexuality academics can be particularly, if ironically, resistant to accepting scientific research related to sexual harassment and their complicity in sexual harassment being a problem in our field.

Conferences tend to provide spaces that, unlike universities, clinics, and institutes, often have no managers, human resource guidelines, or clearly stated sexual harassment policies (though more are developing these). Many events mix the social with the professional and are designed for networking, encouraging junior academics to socialize with senior ones. The mixture of alcohol and more casual attire at conference social events may, for some, further blur the lines between social and professional contexts, further emboldening those who would cross boundaries. To be clear, this is the fault not of casual attire or alcohol, but of those who would or do use them as a pretext to cross boundaries and engage in sexual harassment.

Professional and academic listservs and email discussions are other spaces where sexual harassment may occur. Even with adequate moderation, which is often a minimum that is not met, posts that frequently and ideologically question the legitimacy of research about women and/or minorities undermine the insights this scholarship provides to sexuality research. It also attempts to undermine the researchers themselves who are often women and/or minoritized. It is gender-based, and sometimes an underappreciated form of sexual harassment.

Victims and targets utilize a number of strategies for dissuading a harasser in a professional context. These include moving away to create distance, inviting someone else into a conversation, making broad gestures so someone else's hand must fall away from one's body, and redirecting the conversation. People concerned about the potential for sexual harassment by a colleague may explicitly request meetings in public, ask to leave the door open during meetings, and find ways to avoid one-onone meetings. These are all common tactics used to prevent or end an uncomfortable interaction; however, sexual harassers often ignore these cues. As sexuality researchers, we (should) know that communication is both verbal and nonverbal and that gender scripts should not drive interactions at the expense of some people's autonomy. Western culture does, however, teach men and masculine people to keep going at all costs in the face of "coy" femininity. Social cues are typically quite easy for most people to discern and, when unclear, can be clarified such as by asking if a certain touch or statement was (un)welcome. Verbal communications are usually the last resort, after all clear but ignored physical cues have been given.

The gender breakdown resulting from generations of explicit and implicit gender discrimination means that senior individuals tend to be men, whereas junior people tend to be more mixed. And, in most academic spaces, people of color, working class individuals, first-generation scholars, sexual and gender minorities, and people with disabilities, among other locations, are present in low numbers, if at all, and the isolation can compound the probabilities of sexual harassment (via othering, exoticization, undermining, etc.) and its effects. Like many majority group members, some majority academics may be loath to acknowledge or legitimize the voluminous evidence demonstrating that their positions of power accrue, in part, from discrimination and not only merit, contributing to a culture of downplaying social determinants of success, social location, and thus sexual harassment and its inequities.

\section{Sexual Harassment in Sexuality Research and Professions}

\section{How the "Sex" in Our Fields Can Contribute to a Culture of Sexual Harassment}

As discussed earlier, our field is not immune from the problem of sexual harassment and, we believe, may have certain features that make it ripe for a disproportionate amount of it. We would be remiss if we didn't acknowledge that some of the most wellknown and/or earliest sex researchers have problematic histories attached to them. These histories may have influenced some of the working styles present in our field today. For example, the sexual openness that characterized Alfred Kinsey and his team can be seen in both positive and challenging lights. Kinsey's biographers, using firsthand accounts and oral histories of staff members, described how the interview team spoke openly at work about their personal sex lives, that it was not unusual for the researchers to request personal "sex histories" of those seeking to work at the Institute for Sex Research (as well as their spouses), and that Kinsey asked his research team to keep records of their own personal sexual experiences (e.g., Collins, 1971; Gathorne-Hardy, 1998; Jones, 1997; Pomeroy, 1972; Winther, 1971). Some of Kinsey's biographies also included accounts of sexual behavior occurring between members of the research team (and their spouses) and highlight how some 
may have at times felt maneuvered into such sexual behaviors (Gathorne-Hardy, 1998; Jones, 1997). We feel that the Kinsey team's inclusion of reports about infant and child genital response provided by one or more adults is especially egregious and concerning, for its time and ours.

Kinsey is the most written-about, but other leaders in our field engaged in practices we now see as problematic, including some that were seen as problematic in their time as well. For example, a senior colleague shared that a well-known psychologist (now deceased) would not allow otherwise-enrolled women students to take his classes. Other senior colleagues have recalled (some positively, others negatively) nude massage and sexual behavior occurring openly at sex research, education, and therapy conferences just a few decades ago, and (only negatively) a milieu that was rife with explicit, unwanted sexual attention. Some of our mentors have shared stories about their and others' earlier efforts, within our professional societies, to address sexual harassment.

These important parts of the history of sex research cannot and should not be ignored because, in our view, they are still very present. Our field would do well to grapple with the difficult aspects of researchers' and clinicians' behavior, including - maybe especially — those who are sometimes put on pedestals. Put simply, we wish to address people as whole, complex human beings. We are comfortable admiring some aspects of sex researchers' work while finding other aspects unethical or problematic. We are also comfortable examining our own styles of teaching, researching, practicing, and/or mentoring as we collectively create the kinds of professional communities we hope will engage new generations of bright, creative scholars.

Our field should consider whether or how some of our sex research or clinician "forefathers" used their power to gain sexual access and the ways this has influenced present behavior in our field. To what extent have some people used the sex researcher card to gain sexual access, whether to students, colleagues, or even research subjects? Let's continually consider who we are and what standards make sense for our offices and laboratories. For example, one of us had the experience of a faculty member requiring students to disclose their personal sexual behavior. We also know of at least two instances where participants in contemporary Sexual Attitude Reassessment (SAR) workshops were asked to share details about their own personal sexual behaviors; one of us experienced this firsthand. How do we/you feel about this? Decades ago, SARs and even some university-based sexuality classes were often conducted in the nude. SAR participants were sometimes invited to touch, smell, and/or taste one another. Irvine (2005) chronicled this history as well as the pushback, led largely by women sexologists, against such required experiences, in her book Disorders of Desire: Sexuality and Gender in Modern American Sexology.

Working in sex research or in sexuality professions (therapy, counseling, education, etc.) does indeed require some level of sexual openness and comfort. Sexual liberalism might inhere more openness to sexuality, but it also can implicitly inhere an expectation of sexual expression. For example, a predominant assumption within sexuality research is that sexuality is natural, good, and/or desirable, what many asexual people have identified as "the sexual assumption." In this way, the belief that sexuality is natural can sometimes translate into the belief that people should be open to any sexual opportunity (e.g., If you are sexually liberated, you'd be sexual with me!). But our professional sexuality spaces are about our work, not our personal sexualities. In the same way, we would not expect that people who study aggression or treat people with aggression problems should be open to anyone's expression of aggression, the expectation that any sexuality researcher or professional should be generally open to sexual advances at a sexuality-related conference is misguided and naïve. As we noted above, this belief, that a sexuality researcher must be open to sexual advances and opportunities, may have been facilitated by the precedents set by earlier workplace conduct of certain celebrated sexuality researchers.

\section{Experiences of Sexual Harassment in Our Field}

Too often, sexual harassment is seen only in its most egregious enactments, like those that involve sexual assault or threats. But sexual harassment takes many forms. Indeed, some of the forms people might misinterpret as "minor" can be uniquely devastating, because victims and targets end up questioning their own experiences and thereby access or receive less social support to work through them. As noted earlier, individuals within our field have long shared with one another, through informal networks, their experiences of sexual harassment. This includes information passed down from faculty to graduate students, as when we warn our trainees and new colleagues to be on guard for harassment from particular senior members (nearly all of the examples of which have been, for us, men). It also includes the other direction, from trainees and early career people (largely women) communicating harassment experiences to more established people (mostly women) who may be recognized as feminists in the field. For example, at one recent conference, eight students and early career sexuality researchers came individually to one of us to share their experiences of sexual harassment at that conference. It can include horizontal sharing among groups of women, as when one of us was part of a circle of five women (then all graduate students) who were all sexually propositioned by the same heterosexual senior man in a professional context, while this man never discussed any professional or research issues with the women. Sometimes graduate students warn others of certain faculty known to sexually harass students, along with advice about how to best manage and/or endure the sexual harassment.

Sometimes the harassment itself takes place within social settings like cocktail hour, where discussions about one's research are mere covers for sexual intentions. One of us, as a graduate 
student, watched as senior men denigrated a senior woman's appearance for looking too much like a "grandmother," and then was met with stares upon asking what was bad about looking like a grandmother anyway. Established faculty, women like us or otherwise, are also not immune to the experiences of sexual harassment within our fields. Some mid-career women have reported experiencing harassing comments and behavior when they were considered for senior and leadership positions within their societies, such as reference to rising the ranks more on the basis of their "sex appeal" than merit.

Informal networks or "rumor mills" are helpful and important, but they exist when people believe they cannot, without repercussion, directly target sexual harassment itself or its perpetrators. This is rational: people often feel (or know) that coming forward with accusations of sexual harassment can negatively impact their career development in a culture where victims are rarely believed (at best) and consequences to harassers are minimized (if even present). In this Guest Editorial, we are taking the opportunity to talk more openly about the problem so we can create more equitable professional opportunities within our field, and to eliminate sexual harassment—not just mitigate it.

Each of us who co-authored this Guest Editorial has experienced sexual harassment from within our field. Although there are many commonalities, experiences of sexual harassment are as diverse as the people who experience them. Most of the people we know who are not white, heterosexual men have experienced sexual harassment within our field; our students, our students' friends, our friends and colleagues are examples of those who have shared their own stories or whose sexual harassment we witnessed firsthand. These experiences include: a senior heterosexual man slowly and pointedly looking an early career woman up and down, settling on her breasts; a senior sexual minority man in a position of power kissing an early career sexual minority man without any relational or sexual context; senior heterosexual men giving extensive attention (including long and intimate touches) to conventionally attractive female students and ignoring men and other women; a senior heterosexual man kissing his graduate student, without her interest or consent; a senior sexual minority man offering to include an early career sexual minority man on grants and publications in exchange for oral sex; a senior white heterosexual man cornering a female graduate student in an elevator and kissing her without consent; a senior heterosexual man asking a pregnant researcher how her orgasms changed with pregnancy; and multiple examples of senior men making sexually inappropriate comments to junior career women in public during the Q\&A following their talks or in semi-public at poster sessions. Indeed, most of these examples are so common that we could cite multiple instantiations of them.

The reputations that senior researchers or other professionals acquire from being known sexual harassers can also have further consequences for junior scholars or trainees and for science more generally. These reputations can extend from the harasser to their professional networks, tarnishing the reputations of the harassers' collaborators and trainees. For example, some will make assumptions that their students exchange sexual favors for career advancement, given that their advisor is known to harass students for just this. This can lead to others targeting these students for more harassment, including debasement of their achievements on the basis of these assumptions. Reputations can extend to collaborators and trainees in other ways, too, for example, suggesting that these individuals don't have a problem with (sometimes egregious) sexual harassment.

As sexuality researchers, educators, and clinicians, we can also experience sexual harassment in our public engagements, including with the media. One unfortunately common experience among sexuality researchers is being sexualized by the media, portraying sexuality researchers as "sexy scientists" who embody sexual stereotypes. For example, one of us was described in a book as wearing unprofessional and sexually suggestive clothing in a professional context as a (fabricated) device to entice readers, thereby perpetuating the stereotype. Upon confrontation with this false portrayal, the journalist dismissed the concerns and suggested the portrayal should be flattering. Another of us had a male journalist express deep sexual attractions to a student in our lab as he was interviewing her for an article about the lab research, and she was worried how pushing back would affect the public discussion of her supervisor's lab. Still another of us had a journalist make inferences about our sex life, in print, based on an "off the record" conversation when the interview was over. And, though some sexuality researchers and professionals enjoy being sexy in their style of dress or presentation, some do not, and others are shamed for not meeting the fabricated norm of the "sexy scientist" or "sexy therapist," a norm that can be limited to women in our field. Depending on the context, sexuality researchers may also be desexualized: another of us had the experience of having her breast cleavage digitally erased in a university publication. Shortly thereafter, illustrating the tendency to sexually sensationalize sexuality researchers' and professionals' appearance, she saw a magazine portrayal of her in which the magazine illustrator had greatly enhanced the size of her breasts. That both publications focused, albeit in differing ways, on altering the breasts of a woman scientist is striking.

\section{Consequences of Sexual Harassment for Our Field}

Sexual harassment has serious adverse consequences for us as sexuality researchers and professionals, and for the larger field. In addition to compromising mental and physical health and well-being, sexual harassment minimizes the contributions of people who are already marginalized within the field by sending the message that they are only or primarily valued for their sexuality rather than their expertise or insights or 
are devalued because of their sexuality. Sexual harassment isolates people, shames people, and sometimes makes them question their academic worth. We are individuals who, at times, have questioned our worth as sexuality researchers, educators, or clinicians because of the sexual harassment we have experienced. Each of us has heard from multiple individuals - students, women, sexual minority men, trans individuals, nonbinary individuals, and/or people of color-who told us they stopped coming to sexuality research conferences because of the sexual harassment they experienced. This is a problem for us as faculty mentors, it's a problem for those of us who are responsible to organizations we serve, and it's a problem for any of us who wishes to engage and include strong scientists and talented colleagues in the important work we do. Sexual harassment needs to stop.

\section{Ending Sexual Harassment in Our Field}

\section{Immediate Steps We Can Take to End Sexual Harassment in Our Field}

Fortunately, there are steps we can take to end sexual harassment. The first step is obvious: sexual harassers need to cease their sexual harassment. Too often, ending sexual harassment is seen as incumbent upon victims, which means additional labor put upon those already experiencing victimization. But harassers likely make up a heterogeneous group. Many who sexually harass do so with the conscious intent of causing harm and/or discomfort. These people are unlikely to stop on their own without external pressure, changes in social norms, or consequences. Some people who sexually harass likely do so while ignoring their own doubts about or discomfort with their own behavior, because social scripts (or friends or colleagues) encourage sexually harassing behavior. Some people who are committing acts of sexual harassment may therefore stop with more education and explicit naming of sexual harassment in all its forms as a social problem that our professional community seeks to end. Some people may actually be ignorant of the ways their behavior is sexually harassing. Here, the impact of sexual harassment is present even if the intent is not. Again, education and clear articulation of community norms will be useful, so long as community members center the importance on ending sexual harassment and its harms, and not the feelings of those who (even inadvertently) enact it.

A second step is to transform existing, at-risk spaces into ones where sexual harassment is not tolerated. Too often, faculty receive little training in pedagogical strategies or best practices in management, even though many faculty manage lab of research assistants who are often students. Thus, faculty may approach their interactions with their students in much the same way their mentor(s) approached working with them. In many cases, this works well. But in others (as noted earlier), behaviors may be rooted in problematic historical approaches. There are some aspects of history that don't need to be repeated or reproduced; taking stock of one's leadership style and lab culture is key to moving forward in a positive way. We believe that there are opportunities for positive change in our field's structures, including professional societies and journals.

A third step is to create new, safe(r) spaces that open up room for discussion of how to transform more recalcitrant spaces. Most of us have been a part of the Feminist Sexuality Research Receptions one of us has occasionally organized at the International Academy of Sex Research (IASR) annual meetings, and this has been one way to catalyze those of us dedicated to making sexuality research a space where we recognize and address issues around equity. Others, including Ellen Laan and Leonore Tiefer, have held gatherings for feminist sexuality researchers at IASR over recent past decades. Feminist spaces, though a clear improvement on anti-feminist, feministunfriendly, or even feminist-neutral spaces, are not enough in themselves since they often center questions of women and gender over intersectionality and issues of class, race/ethnicity, sexuality, gender identity, nationality, religiosity, and more. Given that women of color, trans individuals, gender nonconforming people, and others are especially likely to be targets of sexual harassment, we need spaces that are committed to issues of power and equity even within feminism. We need the feminist spaces, and mainstream sexuality research and professional spaces, to be committed to speaking openly and hearing about sexual harassment and its complexities. We also see these spaces, and anti-harassment efforts, as part of broader efforts to create professional communities that attend to feminist values and inclusive principles-for example, supporting childcare sponsorships at conferences (Calisi and a Working Group of Mothers in Science, 2018), letting students bring their infants/ children to class, ensuring access to education and conference spaces for people of a range of disabilities, and making pronoun stickers available for conference badges.

What would education, cultural change, and consequences around reducing or eliminating sexual harassment in sexuality research and professions look like? Below we provide a (non-exhaustive) list of recommendations:

1. Sexual harassers need to cease their sexual harassment. Just stop it. This should go without saying, but perpetrators are often not told that they can and should stop. What does this mean in practice? People who feel the desire to stroke a student's lower back should refrain from doing so. Comments that sexualize non-partner colleagues' or students' bodies (e.g., "All the female graduate students in my department have huge breasts") or link appearance and work (e.g., "Push your boobs up so they'll fund your research") need to stop. Each of these are real examples 
from our own experiences. We understand that many positive, consensual romantic relationships and/or sexual interactions have started at conferences, at work, or even between professors and graduate students. Yet, as sexuality researchers, we also know that there are better, more creative, non-harassing ways to convey romantic or sexual interest than the sexual harassment people so often describe.

2. Comments or "jokes" that a person should have sex with someone else to access funding or professional opportunities need to stop. We know of instances where male mentors and colleagues encouraged women and sexual minority men to engage in sexual behaviors or endure sexual harassment with people who held the key to funding or professional opportunities. Even if intended as a joke, no one should be put in the position of judging whether they might need to engage in unwanted sexual behavior to further their career.

3. Create conversations about codes of conduct and shared values. While it is important to address the kinds of behavior that are unacceptable, it is also important to speak to the kinds of academic and professional environments we wish to create: for example, environments that are intellectually invigorating, civil, respectful, inclusive, and supportive (see Clancy, 2018). Some lab or organizations may choose to articulate these in published Codes of Conduct, or even in aspirational statements, so as to communicate shared values within a group.

4. Reinforce that sexuality research and professions are not about individual colleagues' sexualities. Individuals may sometimes bring their own sexualities into their own work directly or through lived experiences, but this should not be assumed nor should students or professionals be asked to articulate how their personal sexuality relates to their work, as some of us have experienced or seen happen to others. Communications otherwise-including pressure to be "sexually liberal" and thereby receptive to sexual advances-needs to cease. Pressuring colleagues or students to be "sexually open" or not "prude," comments we have repeatedly heard and some of our mentors described as occurring when they were trainees, is a tactic used to coerce sexual access. Openly discussing sexuality can be done without making unconsented-to self-referential comments in lab, research groups, listservs, and at conferences. Using solely colloquial terms-e.g., "jerking off" versus "masturbation"-may also personalize sexuality when done for no professional reason. This is not a call to respectability politics (always a concern for our field) nor is it to preclude people from discussing their own sexuality when others are interested and the circumstances are appropriate. We simply suggest that we all (again, including ourselves in this) be mindful of our language and intentions.

5. Sexuality research and professional organizations need to develop sexual harassment policies that include clear guidelines, reporting policies, and articulated consequences. As sexual harassment disproportionately affects women, people of color, and/or sexual and gender minority individuals (see National Academies of Sciences, Engineering, and Medicine, 2018), we believe these individuals should make up a majority of the committees that are charged with drafting such policies or other ways of addressing harassment. These policies should apply to face-to-face interactions as well as online communications, should be voted on and adopted into societal bylaws, procedures, and records, and must be fully accessible to members (e.g., on society websites, in conference programs and apps). And, these policies need to be followed; continued inaction will only empower harassers. It may seem paternalizing, as adults, to have "professional behavior" explained to us, but the sexual harassment many sexuality researchers and professionals perpetrate starkly illustrates just how needed this guidance is. Indeed it is paternalizing to be told that these policies are not needed, when our and many others' experiences show that they clearly are. That said, we also know that targets rarely report, and for many reasons; thus, we don't see policies as the "answer" to sexual harassment, but just as one piece that should be in place as we change the culture of our academic and professional communities.

6. Anonymous questionnaires and conference evaluations should include questions about experiences of sexual harassment as well as intersecting issues such as experiences of racism or ableism. Conferences routinely include some sort of evaluative materials, and it is important that these address ongoing problems beyond merely technical issues. Societies might also evaluate other aspects of their work such as listserv climate in regard to sexual harassment or sexist comments or behavior, and should share aggregated, anonymized findings to members through usual communication channels (e.g., listserv postings, annual business meeting). It is critical, however, that this is done with expertise, recognizing that some individuals are more empowered to share, even anonymously, than others.

7. Expand opportunities for professional development. Sexual harassment may be more likely to occur in workplaces that are rife with other uncivil or disrespectful behaviors. Thus, universities, clinics, and professional societies might consider making trainings or workshops available on civility promotion programs, de-escalation, or on creating inclusive, supportive workplaces (Clancy, 2018). 
8. As a matter of routine, share organizational policies and reporting mechanisms. Professors should include sexual harassment, assault, and/or misconduct policies and reporting mechanisms on their syllabus; in the U.S., some guidance may be offered from campus Title IX offices or other university policies. Within research or clinical groups, clarify to students, lab managers, postdoctoral fellows, colleagues, and anyone else what the sexual harassment policy at your organization is, how you support it, and what people should do should when policies are violated. At the 2018 annual meeting of the American Association of Sexuality Educators, Counselors, and Therapists, some of us led an effort to include such guidelines in the printed program and on our app; these were also highlighted in the letter from the conference co-chairs.

9. Recognize that sexual harassment can happen anywhere; at social events, during poster sessions, in coffee breaks, on listservs, in private meetings, during meals, etc. Often, the most common advice is to avoid private or hotel room meetings; however, some important events can take place in these spaces. We do discourage asking for or going to private meetings separate from public spaces, as these spaces could be particularly difficult to navigate if sexual harassment does happen, but the trope of private spaces being assault-prone seems to prevent people from realizing that sexual harassment is still happening during public events. So, though physical location can affect the ability to mitigate sexual harassment, we want to remind our colleagues that the behavior is the problem, not the location.

10. Name sexual harassment. If you see a colleague engaging in inappropriate or "creepy" behavior, name what the person is doing in the moment or afterward. The harasser is usually subjecting another person to the sexual harassment because that person holds (or is seen to hold) less power than the harasser or the harasser would like to undermine the power the target does hold. Intervening in this dynamic is important to the target (communicating that they and the behavior are being seen), to bystanders (communicating that harassment will not be tolerated), and to the harasser (putting them on notice). Although we use strong language in describing these processes, we are not actually calling for people to shout "That's sexual harassment!" There are many ways to intervene, some of them educational, some of them humorous, but all of them critical to ending sexual harassment. If you see someone comment on a young woman's appearance, you can say "Hey, I think we should hear about her research." If you see someone touching someone else in inappropriate ways, you can say "Wow, handsy today? Are we just stroking strangers now?" If you hear someone exoticizing a person of color, you can interrupt with "Why are you bringing up racist stereotypes? Let's talk about their work." If you see a student uncomfortably reciprocating sexual flirtations you can politely ask the student "Do you want to come walk with me to speak to that other researcher about their work?" The person can always say no but our experience tells us that our guts are often correct and people are often grateful for a nonconfrontational way to escape these kinds of situations. You have our permission to make fun of these examples, certainly, but only if you come up with your own.

11. Model appropriate behavior. Do not engage in sexualizing, lewd, and/or sexist comments or acts. This should, but clearly does not, go without saying, as does our repeated clarification that sexual harassment is not the same as any attempt at initiating sexual interactions, talking about sex research or therapy, etc., or humor (which is only humor, remember, when all parties find it so).

12. Educate yourself about enthusiastic consent and put this into practice. Again, we acknowledge and appreciate that many meaningful friendships, romantic relationships, and sexual connections were first formed in academic spaces. People can flirt and assess others' romantic or sexual interest; indeed, assessing others' interest is a major aspect of flirting. But do pay attention to social cues and-if you're not sure your comments or behaviors are welcome-ask or step back.

13. Remember: Sexist acts are not just sexualizing but reduce people to their gender or sex. This can include, for example, only asking new mothers about their babies, pregnancies, or breastfeeding, in a professional context, to the exclusion of their work or without consent. For example, while some may want to connect with others around breastfeeding, many people want to discuss it with those who have themselves breastfed. Several of us have had our breastfeeding pointed out at conferences (a few of us have even had this happen from the podium speaker), or had men at conferences who were mere acquaintances ask if we were pumping milk while away from our babies. One male acquaintance, unforgettably, used hand gestures to illustrate. These conversations may be well-intentioned attempts to connect about parenting but-in isolation, out of context, and by acquaintances rather than friends or colleagues-can have the effect of reducing parents (almost always mothers) to their sex or breastfeeding status. Many people re-entering work spaces after having a baby are eager to talk about their work, particularly if they are worried about being perceived as being on a "mommy track" (i.e., shifting their life priorities away from work), and this is mostly targeted at mothers. Please ask us about our work and follow our lead in whether we want to discuss pregnancy, parenting, or breastfeeding.

14. Take responsibility for your actions. When someone tells you that your behavior toward them is inappropriate, understand that this is their experience, and believe 
them. Do not offer excuses. Then, apologize and move on to a professional, work-related topic. If you need clarification, help defuse the moment by apologizing now and asking for details later. If someone contacts you about your behavior, be thankful they both care enough to act and imagine that you have the potential to change, and apologize regardless of your original intention. If you see yourself in any of the examples we've provided above, contact someone to apologize.

15. Stop participating in the culture of sexual harassment. Some of us have perpetuated the problem by telling students, "He's creepy but if you can put up with his behavior, it's an interesting class." And similarly we have nodded our heads in understanding when colleagues or mentors have told us about someone who touched others inappropriately, "but otherwise does excellent research." We need to take these instances seriously. If we've had students complain about a colleague's sexual comments or advances, let's take it upon ourselves to tell that colleague that we would love to continue recommending their class or training opportunities, but we need some assurance about their ability to create and maintain a professional climate. If someone advises us on how to tolerate a colleague or mentor's harassing behavior, let's address that person's behavior rather than feel like we have to put up with harassment. If someone harasses us or someone we know, let's ask the person to stop. Harassment has continued because, obviously, people harass, but also because too many of us (again, even most of us here) have tolerated it, figured out how to adapt to it, and taught others how to adapt. For many of us, this is how we've become accustomed to navigating and, in some ways, surviving professional spaces. These same skills, however, also inadvertently serve to perpetuate a culture of shame and secrecy, and embolden sexual harassers. Of course, the main responsibility is with those who harass, but we can help by ending a culture that supports or tolerates harassment.

\section{Longer-Term Approaches to Ending Sexual Harassment in Our Field}

Ending sexual harassment within our professional spheres is not a trivial task. We included suggestions above that could be actioned "immediately," however, some will take work and time to implement. Sexuality-related professional societies should allocate funding to people committed to ending sexual harassment to conduct research, develop and then evaluate best practices for policies, and adapt these for organizations, societies, and research groups. Researchers might investigate the development of new methods that would help us all better understand issues related to sexual harassment, whether in our own workplaces or other spaces.
Longer-term approaches should center marginalized groups in their efforts, including people of color, gender and sexual minorities, women of various social locations, and/or students. This means centering these groups in terms of perspectives, representation, and power to enact change toward ending sexual harassment (without asking for unpaid labor). It also means attending to issues, outside of but also related to sexual harassment, about racism, white supremacy, ageism, ableism, transphobia, biphobia, homophobia, misogyny, femmephobia, settler colonialism, postcolonialism, and many more. Mainstream sexuality research, education, and therapy are fields with overrepresentation of white majority individuals. Look, for example, at the board members, conference co-chairs, and journal editors connected to most U.S. and Canadian societies-including some of us and the organizations we belong to or lead. Leaders of sexuality research and professional organizations need to grapple with this and develop ways to remediate it. This includes building deeper, meaningful, and mutually beneficial connections with sexuality research and professional communities of color that already exist, like The Women of Color Sexual Health Network and the Association of Black Sexologists and Clinicians. It also includes providing structured opportunities-like questionnaires and/or interviews - to understand minoritized experiences within our field. We cannot draw more people into an inhospitable place, and it is imperative on us all to make clear what it is about sexuality research and professions that are driving minoritized people out and to work toward spaces that are meaningful for potential and actual sexuality researchers. Professional organizations might also consider surveying those who do not renew their memberships, or who stop attending conferences, to better understand individuals reasons for doing so, as companies and universities sometimes do with exit interviews.

As sexual harassment tends to be marked by an abuse of real or perceived power as well as longstanding power differentials, another long-term approach might be to create opportunities for more people to hold positions of power. We might consider more frequently rotating directors, deans, department chairs, membership on grant review committees, and journal editorships, as most societies already do with term limits for officers and Board members. Ideally, this would lead to more diverse perspectives being reflected, as well as more people having the ability to demonstrate national and/or international recognition, which is often key to tenure and promotion decisions. By proactively making power temporary, we might cultivate a sense of more people with power rather than power being primarily placed in a few individuals and potentially "forever." This could have a positive ripple effect with sexual harassment issues. A strategy recommended in the National Academies of Sciences, Engineering, and Medicine Consensus Study Report on the Sexual Harassment of Women has to do with diffusing power structures (National Academies of Sciences, Engineering, and Medicine, 2018). In practice, this may involve 
utilizing mentoring networks rather than individual mentors, group rather than individual advising, and pooled funds in a department for graduate students rather than funds coming from relationships between principal investigators and their students.

\section{How the "Sex" in Sexuality Research and Professions Can Also Contribute to a Culture of Ending Sexual Harassment}

We cannot list every instance of sexual harassment or uncomfortable sexual attention that we, or people we know, have experienced, much less experiences of people we haven't heard from. We know that our failure to include or know about the sexual harassment experiences of our colleagues and students of social locations we don't share might lead to further feelings of isolation and frustration. We hope our sincerity and seriousness in working and being resources with people of any social location who have experienced sexual harassment comes through regardless. As we move to open this conversation, we include ourselves in that process and target group, as a work-in-progress toward ending sexual harassment.

We conclude by noting that, though the "sex" in our fields contributes to the particular challenges of sexual harassment in our field, the "sex" in our fields also offers us uniquely promising opportunities for change. We already discuss sexuality and are comfortable doing so- this is a strength to be capitalized upon. We already navigate personal-professional boundaries around sex by dint of existing in larger culture as sexuality researchers, educators, and clinicians. Many of us are clinicians and have the skills available to guide on best practices for professional conduct in regard to sexuality. Many of us are researchers and can contribute to evaluating best practices, as well as developing new and relevant methodologies and measures for research related to sexual harassment broadly as well as specifically in our field. We are committed to authentic, positive, engaged, and meaningful sexualities. We can use our unique and valuable strengths as sexuality researchers and professionals to work toward ending sexual harassment in our field; please join us.

Acknowledgements Authorship listings are always a challenge, as ordinal listings don't do justice to how papers unfold-who comes up with initial idea(s), who has time or energy to write at a given moment, how the ideas evolve as they bounce among co-authors, and all the emails, outlines, conversations, and edits that move a paper forward. We benefitted from a collaborative process.

\section{References}

Calisi, R. M., \& A Working Group of Mothers in Science. (2018). How to tackle the childcare-conference conundrum. Proceedings of the National Academy of Sciences of the United States of America, 115(12), 2845-2849.

Clancy, K. (2018). Ok, so a few first thoughts on how to handle this. Leadership has to give a shit and want to try something different than policy. Prof societies can fund conflict engagement \& bystander intervention trainings. Prof societies can run workshops on creating lab codes of conduct. Retrieved from https://twitter.com/KateClancy /status/1044585969775599616. Accessed 01 Dec 2018.

Clancy, K. B. H., Nelson, R. G., Rutherford, J. N., \& Hinde, K. (2014). Survey of academic field experiences (SAFE): Trainees report of harassment and assault. PLOS ONE, 9(7), e102172. https://doi. org/10.1371/journal.pone.0102172.

Collins, D. C. (1971). An oral history interview with Dorothy Craig Collins/Interviewer: James H. Jones [71-053]. Bloomington: Center for Documentary Research and Practice, Indiana University.

Dzau, V. J., \& Johnson, P. A. (2018). Ending sexual harassment in academic medicine. New England Journal of Medicine, 379, 1589-1591. https://doi.org/10.1056/NEJMp1809846.

Flaherty, C. (2018). Sociology facing harassment 'rumors' defers award. Inside Higher Ed. Retrieved from https://www.insidehigh ered.com/quicktakes/2018/08/06/sociologist-facing-harassment -rumors-defers-award. Accessed 01 Dec 2018.

Gathorne-Hardy, J. (1998). Sex the measure of all things: A life of Alfred $C$. Kinsey. Bloomington: Indiana University Press.

Grollman, E. (2018). A call for sociology's \#MeToo mo(ve)ment. Retrieved from https://egrollman.com/2018/08/06/metoo-socio logy/. Accessed 01 Dec 2018.

Irvine, J. M. (2005). Disorders of desire: Sexuality and gender in modern American sexology. Philadelphia, PA: Temple University Press.

Jones, J. H. (1997). Alfred Kinsey: A public/private life. New York: W.W. Norton \& Company.

Mondon, M. (2018). Penn professor resigns following allegations of inappropriate relationships with students. Philly Voice. Retrieved from https://www.phillyvoice.com/penn-professor-kurzbanresigns-sexual-misconduct-allegations-relationships-students/. Accessed 01 Dec 2018.

National Academies of Sciences, Engineering, and Medicine. (2018). Sexual harassment of women: Climate, culture, and consequences in academic sciences, engineering, and medicine. Washington, DC: The National Academies Press. https://doi.org/10.17226 124994.

Pomeroy, W. B. (1972). Dr. Kinsey and the institute for sex research. New Haven, CT: Yale University Press.

Schulz, V. (2018). Reconceptualizing sexual harassment, again. Yale Law Journal Forum, 128, 22-66.

Winther, M. G. (1971). An oral history interview with Mary G. Winther/ Interviewer: James H. Jones [71-008]. Bloomington: Center for Documentary Research and Practice, Indiana University.

Publisher's Note Springer Nature remains neutral with regard to jurisdictional claims in published maps and institutional affiliations. 